\begin{tabular}{|c|c|c|c|c|c|c|}
\hline \multirow{4}{*}{ Impact Factor: } & ISRA (India) & $=3.117$ & SIS (USA) & $=0.912$ & ICV (Poland) & $=6.630$ \\
\hline & ISI (Dubai, UAE & $=0.829$ & РИНЦ (Russia) & $=0.156$ & PIF (India) & $=1.940$ \\
\hline & GIF (Australia) & $=0.564$ & ESJI $(\mathrm{KZ})$ & $=8.716$ & IBI (India) & $=4.260$ \\
\hline & JIF & $=1.500$ & SJIF (Morocco) & $=5.667$ & OAJI (USA) & $=0.350$ \\
\hline
\end{tabular}

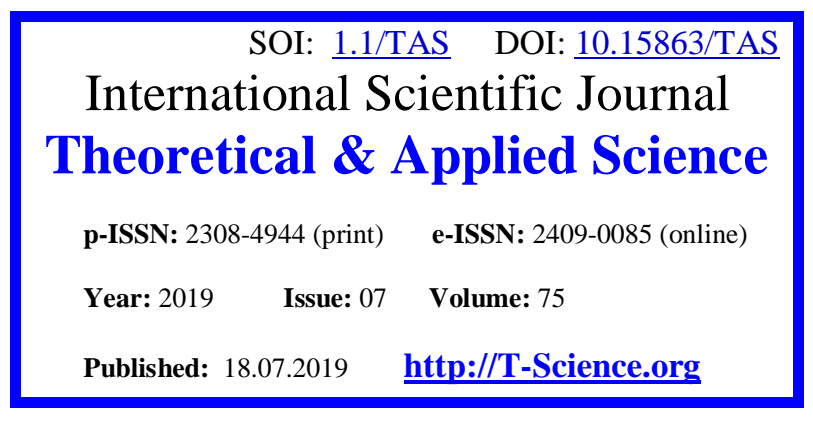

QR - Issue

QR - Article

International Scientific Journal
Theoretical \& Applied Science

p-ISSN: 2308-4944 (print) e-ISSN: 2409-0085 (online)

Published: 18.07.2019 http://T-Science.org
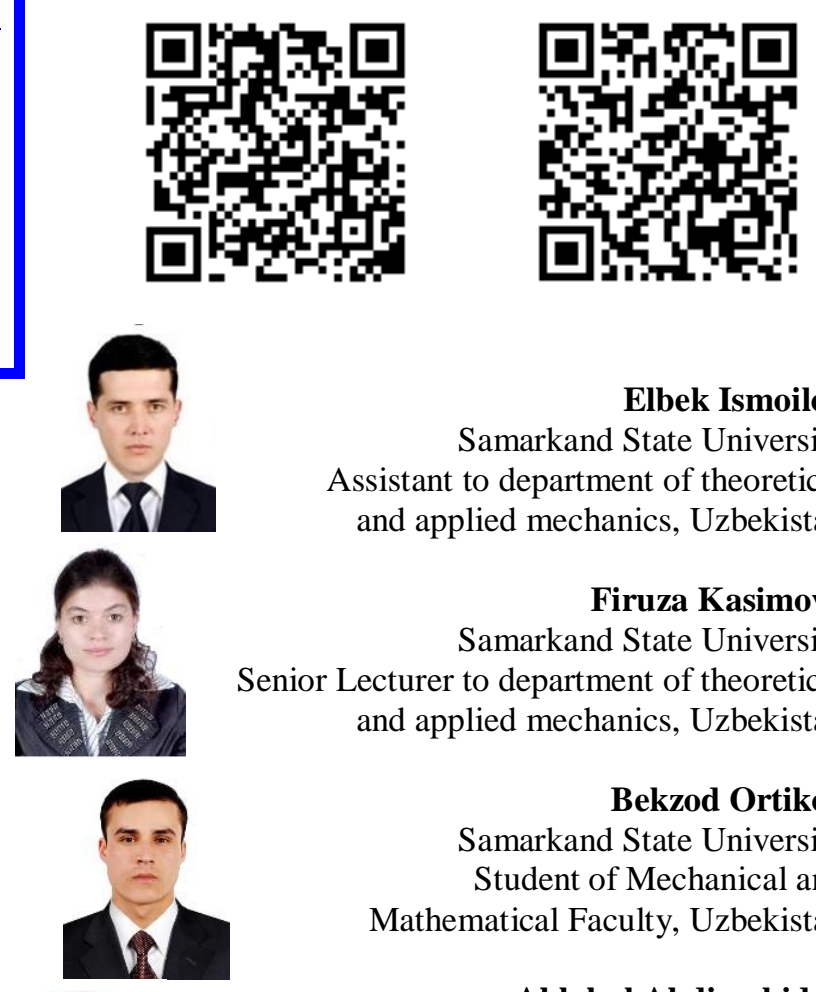

Elbek Ismoilov

Samarkand State University

Assistant to department of theoretical and applied mechanics, Uzbekistan

Firuza Kasimova

Samarkand State University Senior Lecturer to department of theoretical and applied mechanics, Uzbekistan

Bekzod Ortikov

Samarkand State University

Student of Mechanical and Mathematical Faculty, Uzbekistan

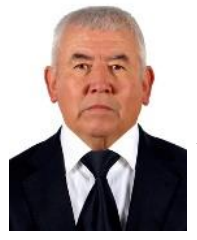

Ablakul Abdirashidov

Samarkand State University

Corresponding member of International Academy, Doctor of Physical and Mathematical

Sciences, Docent to department of theoretical and applied mechanics, Uzbekistan,

\title{
APPLICATION OF ADOMIAN DECOMPOSITION METHOD TO SOLVING OF SOME BOUNDARY PROBLEMS MATHEMATICAL PHYSICS
}

Abstract: In this paper, Adomian decomposition method has been applied to obtain particular solution of some boundary problems for the equations of mathematical physics. It is shown that this method are effective and more powerful mathematical tools for the solution of the partial differential equations.

Key words: particular solution, boundary problem, heat dissipation equation, wave equation, Adomian decomposition method.

Language: Russian

Citation: Ismoilov, E., Kasimova, F., Ortikov, B., \& Abdirashidov, A. (2019). Application of Adomian decomposition method to solving of some boundary problems mathematical physics. ISJ Theoretical \& Applied Science, 07 (75), 201-205.

Soi: http://s-o-i.org/1.1/TAS-07-75-34 Doi: crossef https://dx.doi.org/10.15863/TAS.2019.07.75.34

Classifiers: Theoretical research in mathematics.

\section{ПРИМЕНЕНИЕ МЕТОДА РАЗЛОЖЕНИЯ АДОМИАНА К РЕШЕНИЮ НЕКОТОРЫХ КРАЕВЫХ ЗАДАЧ МАТЕМАТИЧЕСКОЙ ФИЗИКИ}

Аннотация: В данной работе метод разложения Адомиана применены для нахождения частных решений некоторых краевых задач для уравнение математической физики. Показано, что это метод являются эффективными и более мощчными математическими инструментами для решения дифференциальных уравнений в частных производных.

Ключевые слова: частное решение, краевая задача, уравнения теплопроводности, волновое уравнение, метод разложения Адомиана. 


\begin{tabular}{llllll} 
& ISRA (India) $=\mathbf{3 . 1 1 7}$ & SIS (USA) & $=\mathbf{0 . 9 1 2}$ & ICV (Poland) & $=\mathbf{6 . 6 3 0}$ \\
Impact Factor: & ISI (Dubai, UAE) $=\mathbf{0 . 8 2 9}$ & PUHЦ (Russia) $=\mathbf{0 . 1 5 6}$ & PIF (India) & $=\mathbf{1 . 9 4 0}$ \\
& GIF (Australia) $=\mathbf{0 . 5 6 4}$ & ESJI (KZ) & $=\mathbf{8 . 7 1 6}$ & IBI (India) & $\mathbf{4 . 2 6 0}$ \\
JIF & $=\mathbf{1 . 5 0 0}$ & SJIF (Morocco) $=\mathbf{5 . 6 6 7}$ & OAJI (USA) & $\mathbf{0 . 3 5 0}$ \\
\hline
\end{tabular}

Введение.

Явные решения математического моделирования физико-механических задач имеют фундаментальное значение. Линейные и нелинейные уравнения в частных производных встречаются при описании многих процессов и явлений в физике, механике, биологии, химии и т.д. Решение краевых задач с такими уравнениями в частных производных являются одной их основных проблем математической физики и инженерных наук. За прошлые несколько десятилетий математики и физики сделали значительные успехи в этом направлении $[6,8,9$, 10]. Многие из этих уравнений не имеют точных аналитических решений. С другой стороны, решение этих нелинейных уравнений аналитически могут вести некоторые авторы, которые глубоко знают описание некоторых физических процессов. В результате эти уравнения должны быть решены, используя другие методы. Последние годы были разработаны различные методики решению таких уравнений, например, метод гомотопического анализа [1], метод вариационных итераций (МВИ) $[5,6]$, метод разложения Адомиана (MPA) $[3,6]$, метод гомотопического возмущения $[4,6]$, упрощенный метод укороченных разложений [2, 7, 8] и др., а также их различные модифицированные варианты $[6,8]$. В данной работе метод разложения Адомиана применен для нахождения частных решений некоторых краевых задач для уравнений математической физики.

\section{Постановка задачи.}

Рассмотрим линейное дифференциальное уравнение в частных производных, то есть уравнения теплопроводности:

$$
u_{t}(x, t)=a^{2} u_{x x}(x, t)+f(x, t),
$$

$0<x<l, t>0$

или волнового уравнения

$$
u_{t t}(x, t)=a^{2} u_{x x}(x, t)+f(x, t)
$$

$0<x<l, t>0$

и граничных условие (задача Дирихле):

$$
u(0, t)=\varphi(t), u(l, t)=\psi(t)
$$

или (задача Неймана):

$$
u_{x}(0, t)=\mu(t), u_{x}(l, t)=v(t) .
$$

где $f(x, t), \varphi(t), \psi(t), \mu(t), v(t)$ - известные функции; $u(x, t)$ - искомая функция.

\section{Алгоритм метода разложения Адомиана.}

Нелинейное дифференциальное уравнение в частных производных перепишем в виде

$$
L u(x, t)=q(x, t)-N u(x, t),
$$

где $L$ - дифференциальный оператор; $L^{-1}-$ интегральный оператор.
Применение обратного оператора к заданному уравнению дает соотношение вида:

$$
u(x, t)=f(x, t)-L^{-1}[N u(x, t)] .
$$

Основная идея МРА это составление функционального уравнения вида

$$
u(x, t)=\sum_{n=0}^{\infty} u_{n}(x, t) .
$$

Отсюда имеем рекуррентное соотношение вида [6]:

$$
u_{0}(x, t)=f(x, t) ; \quad u_{n+1}=-L^{-1}\left[N u_{n}(x, t)\right]
$$

, $n \geq 0$.

\section{Пример 1.}

Найти решение следующей краевой задачи с уравнением теплопроводности в виде:

$$
\begin{aligned}
& u_{t}=u_{x x}+\sin x, 0<x<\pi, t>0, \\
& u_{x}(0, t)=1, \quad u_{x}(\pi, t)=-1 .
\end{aligned}
$$

Сначала введем следующие обозначения: $u(x, 0)=f(x)$.

Метод разложения Адомиана.

$$
\int_{0}^{t} u_{\xi}(x, \xi) d \xi=\int_{0}^{t}\left[u_{x x}(x, \xi)+\sin x\right] d \xi \text {, }
$$

отсюда имеем

$$
u(x, t)=f(x)+t \sin x+\int_{0}^{t} u_{x x}(x, \xi) d \xi .
$$

По идее МРА:

$$
u(x, t)=\sum_{n=0}^{\infty} u_{n}(x, t) .
$$

Исходя из этого имеем

$$
\begin{aligned}
& u_{0}+u_{1}+u_{2}+\ldots=f(x)+t \sin x+ \\
& +\int_{0}^{t}\left[u_{0}+u_{1}+u_{2}+\ldots\right]_{x x} d \xi
\end{aligned}
$$

и

$$
\begin{gathered}
u_{0}=f(x)+t \sin x \\
u_{1}=\int_{0}^{t}\left[u_{0}\right]_{x x} d \xi=t f^{\prime \prime}(x)-\frac{t^{2}}{2 !} \sin x \\
u_{2}=\int_{0}^{t}\left[u_{1}\right]_{x x} d \xi=\frac{t^{2}}{2 !} f^{I V}(x)+\frac{t^{3}}{3 !} \sin x ; \ldots \\
u_{n}=\int_{0}^{t}\left[u_{n-1}\right]_{x x} d \xi=\frac{t^{n}}{n !} f^{(2 n)}(x)+ \\
+(-1)^{n} \frac{t^{n+1}}{(n+1) !} \sin x
\end{gathered}
$$




\begin{tabular}{llllll} 
& ISRA (India) $=3.117$ & SIS (USA) & $=\mathbf{0 . 9 1 2}$ & ICV (Poland) & $=\mathbf{6 . 6 3 0}$ \\
Impact Factor: & ISI (Dubai, UAE) $=\mathbf{0 . 8 2 9}$ & PUHIL (Russia) $=\mathbf{0 . 1 5 6}$ & PIF (India) & $=\mathbf{1 . 9 4 0}$ \\
GIF (Australia) & $=\mathbf{0 . 5 6 4}$ & ESJI (KZ) & $=\mathbf{8 . 7 1 6}$ & IBI (India) & $\mathbf{4 . 2 6 0}$ \\
& JIF & $=\mathbf{1 . 5 0 0}$ & SJIF (Morocco) $=\mathbf{5 . 6 6 7}$ & OAJI (USA) & $\mathbf{0 . 3 5 0}$ \\
\hline
\end{tabular}

и т.д. Окончательно получим решение задачи вида $u(x, t)=u_{0}+u_{1}+\ldots=$

$=\sum_{k=0}^{\infty}\left[\frac{t^{k}}{k !} f^{(2 k)}(x)+(-1)^{k} \frac{t^{k+1}}{(k+1) !} \sin x\right]=$

$=\sum_{k=0}^{\infty} \frac{t^{k}}{k !} f^{(2 k)}(x)+\sin x\left[1-\sum_{k=0}^{\infty} \frac{(-t)^{k}}{k !}\right]=$

$=\sum_{k=0}^{\infty} \frac{t^{k}}{k !} f^{(2 k)}(x)+\left[1-e^{-t}\right] \sin x$.

Теперь неизвестную функцию $f(x)$ найдем из условие (2). В результате имеем

$$
u_{x}(0, t)=1=\sum_{k=0}^{\infty} \frac{t^{k}}{k !} f^{(2 k+1)}(0)+1-e^{-t}
$$

и

$$
u_{x}(\pi, t)=-1=\sum_{k=0}^{\infty} \frac{t^{k}}{k !} f^{(2 k+1)}(\pi)-1+e^{-t} .
$$

или

$$
\sum_{k=0}^{\infty} \frac{t^{k}}{k !} f^{(2 k+1)}(0)=\sum_{m=0}^{\infty} \frac{(-1)^{m} t^{m}}{m !}
$$

и

$$
\sum_{k=0}^{\infty} \frac{t^{k}}{k !} f^{(2 k+1)}(\pi)=\sum_{m=0}^{\infty} \frac{(-1)^{m+1} t^{m}}{m !} .
$$

Из первого равенства (5) имеем

$$
f^{\prime}(0)=1 ; \quad f^{\prime \prime \prime}(0)=-1 ; \quad f^{V}(0)=1 ; \ldots ;
$$$$
f^{(2 n+1)}(0)=(-1)^{n} \text { и т.д. }
$$

Из второго равенства (5) имеем

$$
\begin{aligned}
& f^{\prime}(\pi)=-1 ; \quad f^{\prime \prime \prime}(\pi)=1 ; \quad f^{V}(\pi)=-1 ; \ldots ; \\
& f^{(2 n+1)}(0)=(-1)^{n+1} \text { и т.д. }
\end{aligned}
$$

В общем случае, найти функцию $f(x)$, удовлетворяющий условий (6) и (7), иногда невозможно. В частном случае, функцию $f(x)$ ищем в виде

$$
f(x)=\sum_{k=0}^{\infty} a_{k} x^{k}
$$

Коэффициентов этого ряда $a_{k}$ находим из условий (6) и (7), а из условие (6) имеем

$$
a_{1}=1 ; \quad a_{3}=\frac{-1}{3 !} ; \ldots ; \quad a_{2 n+1}=\frac{(-1)^{n}}{(2 n+1) !} \text { и }
$$

т.д.

$$
\begin{aligned}
& \text { Отсюда } \\
& f(x)=\sum_{k=0}^{\infty} a_{2 k} x^{2 k}+\sin x,
\end{aligned}
$$

а из условия (7) имеем

$$
f^{\prime}(\pi)=\sum_{k=1}^{\infty} 2 k a_{2 k} \pi^{2 k-1}-1=-1 .
$$

Отсюда

$$
\begin{gathered}
\sum_{k=1}^{\infty} 2 k a_{2 k} \pi^{2 k-1}=0=\sin \pi=\sum_{m=1}^{\infty} \frac{(-1)^{m-1} \pi^{2 m-1}}{(2 m-1) !} \\
\text { Отсюда верно, что } a_{2 k}=\frac{(-1)^{k}}{(2 k) !}, k=1,2, \ldots . \text { или } \\
a_{2 k}=0, k=1,2, \ldots \text { Если } a_{2 k}=0, k=1,2, \ldots, \text { то }
\end{gathered}
$$
получим стационарное решение вида $u(x, t)=u(x)$ . Этот случай более прост. Поэтому самый интересный случай, когда $a_{2 k}=\frac{(-1)^{k}}{(2 k) !}, k=1,2, \ldots$, при этом получим

$$
f(x)=a_{0}-1+\cos x+\sin x
$$

или

$f(x)=\cos x+\sin x$.

Соответственно к этому имеем решение $u(x, t)$ в виде:

$$
\begin{aligned}
& u(x, t)=\sum_{k=0}^{\infty} \frac{t^{k}}{k !} f^{(2 k)}(x)+\left[1-e^{-t}\right] \sin x= \\
& =e^{-t} \cos x+\sin x .
\end{aligned}
$$

\section{Пример 2.}

Найти решение следующей краевой задачи с волновым уравнением в виде:

$$
u_{t t}=u_{x x}+\sin x, \quad 0<x<\frac{\pi}{2}, t>0,
$$

(9)

$$
u(0, t)=0, \quad u_{x}\left(\frac{\pi}{2}, t\right)=0 .
$$

Сначала введем следующие обозначения:

$$
u(x, 0)=\varphi(x), u_{t}(x, 0)=\psi(x),
$$

где $\varphi(x), \psi(x)$ - пока неизвестные функции.

Метод разложения Адомиана.

$$
\begin{aligned}
& \int_{0}^{t} d \eta \int_{0}^{\eta} u_{\xi \xi}(x, \xi) d \xi= \\
& =\int_{0}^{t} d \eta \int_{0}^{\eta}\left[u_{x x}(x, \xi)+\sin x\right] d \xi,
\end{aligned}
$$

отсюда имеем

$$
\begin{aligned}
& u(x, t)=\varphi(x)+t \psi(x)+\frac{t^{2}}{2 !} \sin x+ \\
& +\int_{0}^{t} d \eta \int_{0}^{\eta} u_{x x}(x, \xi) d \xi
\end{aligned}
$$

По идее МРА: 


\begin{tabular}{|c|c|c|c|c|c|c|}
\hline \multirow{4}{*}{ Impact Factor: } & ISRA (India) & $=3.117$ & SIS (USA) & $=0.912$ & ICV (Poland) & $=6.630$ \\
\hline & ISI (Dubai, UAE & $=0.829$ & РИНЦ (Russia) & $=0.156$ & PIF (India) & $=1.940$ \\
\hline & GIF (Australia) & $=0.564$ & ESJI (KZ) & $=8.716$ & IBI (India) & $=4.260$ \\
\hline & JIF & $=1.500$ & SJIF (Morocco & $=5.667$ & OAJI (USA) & $=0.350$ \\
\hline
\end{tabular}

$$
u(x, t)=\sum_{n=0}^{\infty} u_{n}(x, t) .
$$

Исходя из этого имеем

$$
\begin{aligned}
& u_{0}+u_{1}+u_{2}+\ldots=\varphi(x)+t \psi(x)+\frac{t^{2}}{2 !} \sin x+ \\
& +\int_{0}^{t} d \eta \int_{0}^{\eta}\left[u_{0}+u_{1}+u_{2}+\ldots\right]_{x x} d \xi \\
& \text { и }
\end{aligned}
$$

$$
\begin{aligned}
& u_{0}=\varphi(x)+t \psi(x)+\frac{t^{2}}{2 !} \sin x ; \\
& u_{1}=\int_{0}^{t} d \eta \int_{0}^{\eta}\left[u_{0}\right]_{x x} d \xi= \\
& =\frac{t^{2}}{2 !} \varphi^{\prime \prime}(x)+\frac{t^{3}}{3 !} \psi^{\prime \prime}(x)-\frac{t^{4}}{4 !} \sin x \\
& u_{2}=\int_{0}^{t} d \eta \int_{0}^{\eta}\left[u_{1}\right]_{x x} d \xi= \\
& =\frac{t^{4}}{4 !} \varphi^{I V}(x)+\frac{t^{5}}{5 !} \psi^{I V}(x)+\frac{t^{6}}{6 !} \sin x \\
& ; \ldots ; ; \\
& u_{n}=\int_{0}^{t} d \eta \int_{0}^{\eta}\left[u_{n-1}\right]_{x x} d \xi=\frac{t^{2 n}}{(2 n) !} \varphi^{(2 n)}(x)+ \\
& +\frac{t^{2 n+1}}{(2 n+1) !} \psi^{2 n}(x)+(-1)^{n} \frac{t^{2 n+2}}{(2 n+2) !} \sin x
\end{aligned}
$$

и т.д. Окончательно получим решение задачи вида $u(x, t)=u_{0}+u_{1}+\ldots=$

$=\sum_{k=0}^{\infty}\left[\frac{t^{2 k}}{(2 k) !} \varphi^{(2 k)}(x)+\frac{t^{2 k+1}}{(2 k+1) !} \psi^{(2 k)}(x)\right]+$

$+(1-\cos t) \sin x$.

Теперь неизвестную функцию $f(x)$ найдем из условие (10). В результате имеем

$u(0, t)=0=\sum_{k=0}^{\infty}\left[\frac{t^{2 k}}{(2 k) !} \varphi^{(2 k)}(0)+\frac{t^{2 k+1}}{(2 k+1) !} \psi^{(2 k)}(0)\right]$

и

$$
\begin{aligned}
& u_{x}\left(\frac{\pi}{2}, t\right)=0= \\
& =\sum_{k=0}^{\infty}\left[\frac{t^{2 k}}{(2 k) !} \varphi^{(2 k+1)}\left(\frac{\pi}{2}\right)+\frac{t^{2 k+1}}{(2 k+1) !} \psi^{(2 k+1)}\left(\frac{\pi}{2}\right)\right] .
\end{aligned}
$$

$$
\varphi^{(2 k)}(0)=0 \text { и } \psi^{(2 k)}(0)=0 k=0,1, \ldots
$$

Из второго равенства (12) имеем

$$
\varphi^{(2 k+1)}\left(\frac{\pi}{2}\right)=0 \text { и } \psi^{(2 k+1)}\left(\frac{\pi}{2}\right)=0, k=0,1, \ldots .
$$

В общем случае, найти функции $\varphi(x)$ и $\psi(x)$, удовлетворяющий условий (13) и (14), иногда невозможно. В частном случае, функции $\varphi(x)$ и $\psi(x)$ ищем в виде

$$
\varphi(x)=\sum_{k=0}^{\infty} a_{k} x^{k} \quad \text { и } \psi(x)=\sum_{k=0}^{\infty} b_{k} x^{k} .
$$

Коэффициентов этого ряды $a_{k}$ и $b_{k}$ находим из условий (13) и (14), а из условия (13) имеем

$$
a_{2 k}=0 \text { и } b_{2 k}=0, k=0,1, \ldots \text {. Отсюда }
$$$$
\varphi(x)=\sum_{k=0}^{\infty} a_{2 k+1} x^{2 k+1} \text { и } \psi(x)=\sum_{k=0}^{\infty} b_{2 k+1} x^{2 k+1} \text {, }
$$

а из условия (14) имеем

$$
\varphi^{\prime}\left(\frac{\pi}{2}\right)=\sum_{k=0}^{\infty}(2 k+1) a_{2 k+1}\left(\frac{\pi}{2}\right)^{2 k}=0=\cos \frac{\pi}{2}
$$

и

$$
\psi^{\prime}\left(\frac{\pi}{2}\right)=\sum_{k=0}^{\infty}(2 k+1) b_{2 k+1}\left(\frac{\pi}{2}\right)^{2 k}=0=\cos \frac{\pi}{2} .
$$

Отсюда верно, что

$\varphi(x)=\sin x$ и $\psi(x)=\sin x$.

Соответственно к этому имеем решение $u(x, t)$ в виде:

$$
\begin{aligned}
& u(x, t)=\sum_{k=0}^{\infty}\left[\frac{t^{2 k}}{(2 k) !} \varphi^{(2 k)}(x)+\frac{t^{2 k+1}}{(2 k+1) !} \psi^{(2 k)}(x)\right]+ \\
& +(1-\cos t) \sin x=(1+\sin t) \sin x .
\end{aligned}
$$

\section{Выводы.}

Таким образом, изучены применения метода разложения Адомиана к приближенному решению краевых задач. Результаты сравнены с точным решением краевой задачи и результатом, полученным с помощью математического пакета Maple 17. Из сравнений ясно, что эти метод достаточно точны. Поэтому, эти методы являются мощными математическими инструментами и с их помощью может быть решен большой класс нелинейных краевых задач, используемых в математической физики и инженерных науках $[1$, $2,11,12]$.

Из первого равенства (12) имеем 


\begin{tabular}{llllll} 
& ISRA (India) $=\mathbf{3 . 1 1 7}$ & SIS (USA) & $=\mathbf{0 . 9 1 2}$ & ICV (Poland) & $=\mathbf{6 . 6 3 0}$ \\
Impact Factor: & ISI (Dubai, UAE) $=\mathbf{0 . 8 2 9}$ & PUHL (Russia) $=\mathbf{0 . 1 5 6}$ & PIF (India) & $=\mathbf{1 . 9 4 0}$ \\
& GIF (Australia) $=\mathbf{0 . 5 6 4}$ & ESJI (KZ) & $=\mathbf{8 . 7 1 6}$ & IBI (India) & $=\mathbf{4 . 2 6 0}$ \\
& JIF & $\mathbf{1 . 5 0 0}$ & SJIF (Morocco) $=\mathbf{5 . 6 6 7}$ & OAJI (USA) & $\mathbf{0 . 3 5 0}$ \\
\hline
\end{tabular}

\section{References:}

1. Abdirashidov, A., Ortiqov, B. B., Qadirov, N. X., \& Abdurashidov, A. A. (2018). Exact solution of fractional diffusion equations using the variational iteration method and Adomian decomposition method. International Scientific Journal Theoretical \& Applied Science, 05 (61), pp.101-107.

2. Abdurashidov, A. A., Ortiqov, B. B., Qadirov, N. X., \& Abdirashidov, A. (2018). Exact solution of some nonlinear evolutionary equations using the modified simple equation method. International Scientific Journal Theoretical \& Applied Science, 3(59).

3. Adomian, G. (1994). Solving Frontier Problems of Physics: The Decomposition Method. 1st Edn., Kluwer Academic, Boston.

4. He, J. H. (2003). Homotopy perturbation method: A new nonlinear analytical technique. Applied Math. Comput., 135, pp.73-79.

5. He, J. H., \& Wu, X. H. (2007). Variational iteration method: New development and applications. Comp. Math. Appl., 54, pp.881894.

6. Wazwaz, A. M. (2009). Partial Differential Equations and Solitary Waves Theory. (p.761). Higher Education Press, Berlin Heidelberg.

7. Abdurashidov, A. A. (2018). Tochnoye resheniye nekotorix nelineynix uravneniy Gardnera uproshennim metodom ukorochennix razlojeniy. Mejdunarodniy setevoy nauchnoprakticheskiy jurnal «Nauka sredi nas». Vipusk: 6.

8. Kudryashov, N. A. (2010). Metodi nelineynoy matematicheskoy fiziki. (p.368). Uchebnoye posobiye. 2-ye izd. Dolgoprudniy: Intellekt.

9. Salohiddinov, M. (2002). Matematik fizika tenglamalari. (p.448). Toshkent: O'zbekiston.

10. Bisadze, A. V., \& Kalinichenko, D. F. (1985). Sbornik zadach po uravneniyam matematicheskoy fiziki. (p.310). Ucheb. posobiye dlya mexaniko-matemat. i fiz. spes. vuzov. 2-ye izd., dop. Moskva: Nauka.

11. Abdirashidov, A., Ortikov, B., Kadirov, N., \& Abdurashidov, A. (2019). Application of Adomian decomposition method, Taylor series method and a variational iterations method to solving a second order ordinary differential equations. International Scientific Journal Theoretical \& Applied Science, 05 (73), 1-5.

12. Abdirashidov, A., Karshiyev, A., Ortikov, B., \& Kadirov, N. (2018). Application of approximate Adomian decomposition method and a variational iterations method to solving a cauchy problem with the heat dissipation and Laplace equations. International Scientific Journal Theoretical \& Applied Science, 12 (68), 323329. 